\title{
RINGS OF EQUIVALENT DOMINANT AND CODOMINANT DIMENSIONS
}

\author{
GARY L. EERKES
}

ABSTRACT. Continuing an earlier examination of the codominant dimeno sion of rings and modules, a categorical characterization is given for rings of equivalent dominant and codominant dimensions. Specifically, the ques. tion is reduced to when ${ }_{R} R$ and the left minimal injective cogenerator ${ }_{R}$ u can be used as test modules respectively for the dominant dimension of the projective modules and the codominant dimension of the injective modules. These conditions are in turn characterized by when the injective projective modules are $\mathbf{\Sigma}$-injective. Also, a new and shortened version is given for the proof that the codominant dimension of the injective modules is equal to the dominant dimension of the projective modules.

Throughout, $R$ is an associative ring with unity and all modules considered are unital. We denote a minimal injective cogenerator for ${ }_{R}^{\text {MT }}$ by ${ }_{R} \mathcal{U}$, such being isomorphic to an injective hull of a direct sum of a complete representative class of simple left $R$-modules. For a left perfect ring $R$ and a left $R$-module ${ }_{R} M$ we define the codominant dimension of ${ }_{R} M$, denoted codom $\operatorname{dim}_{R} M$, to be greater than or equal to $n$ if for a minimal projective resolution $\cdots \rightarrow P_{n} \rightarrow \cdots \rightarrow P_{1} \rightarrow M \rightarrow 0, P_{i}$ is injective for $i=1$, $2, \cdots, n$. The left codominant dimesnion of $R$ is defined to be codom $\operatorname{dim}{ }_{R} \mathcal{U}$. The dominant dimension of ${ }_{R} M$ is defined in a dual manner, and the left dominant dimension of $R$ is $\operatorname{dom} \operatorname{dim}_{R} R$. Further, for $R$ left perfect, we define codom $\operatorname{dim}_{R} g$ to be inf $\left\{\right.$ codom $\operatorname{dim}_{R} E:{ }_{R} E$ injective\}. Similarly, dom $\operatorname{dim}_{R} \mathcal{P}$ is given by inf $\left\{\operatorname{dom} \operatorname{dim}_{R} P:{ }_{R} P\right.$ projective $\}$. Finally, we say that ${ }_{R} E\left({ }_{R} P\right)$ is a test module for ${ }_{R} g\left({ }_{R} \mathcal{P}\right)$ provided

$$
\text { codom } \operatorname{dim}_{R} E=\text { codom } \operatorname{dim}_{R} g \quad\left(\operatorname{dom} \operatorname{dim}_{R} P=\operatorname{dom} \operatorname{dim}_{R} \mathcal{P}\right) \text {. }
$$

In [2] we showed that $\operatorname{dom} \operatorname{dim}_{R} \mathcal{P}=$ codom $\operatorname{dim}_{R}$ g. As an application it can 1973.

Presented to the Society, January 25, 1973; received by the editors April 16, 16 A52.

AMS (MOS) subject classifications (1970). Primary 16A60; Secondary 16A50,

Key words and phrases. Perfect rings, injective projective modules, codominant and dominant dimensions, $\Sigma$-injectivity. 
be shown that the left codominant dimension of a left artinian ring is equivalent to the ring's left dominant dimension. In fact, this follows from the result that the minimal injective cogenerator ${ }_{R} U$ and the left regular module ${ }_{R} R$ can be used as test modules for ${ }_{R} \mathfrak{I}$ and ${ }_{R} \mathcal{P}$, respectively. In this paper we propose to give a categorical characterization of those rings for which this can be done.

As shall be seen, this is closely related to when the direct sum of projective injective modules is injective and rings with ascending condition on annihilators (see [3]). To facilitate this discussion we provide the following definitions.

An injective module ${ }_{R} M$ is $\Sigma$-injective provided $\bigoplus \Sigma_{\Omega} M$ is injective for all $\Omega$. Faith [3, Proposition 3.3] has shown that it is sufficient to consider $\Omega$ of countable cardinality in testing for $\Sigma$-injectivity. As the categorical dual concept we have $\pi$-projectivity.

For a left $R$-module ${ }_{R} M$ and a subset $X \subseteq M$ we set

$$
\operatorname{Ann}_{R}(X)=\{r \in R: r X=0\} \text {. }
$$

The collection of all such left ideals for a given ${ }_{R} M$ is denoted by $\mathfrak{Q n n n}_{R}(M)$. For the special case of ${ }_{R} M={ }_{R} R, \operatorname{Qnn}_{R}(R)$ is called the set of left annulets of $R$.

1. The left regular module ${ }_{R} R$ as a test module. In this section we determine when ${ }_{R} R$ is a test module. Clearly this is a triviality when dom $\operatorname{dim}_{R} R=0$.

Theorem 1.1. Let $R$ be a left perfect ring of positive left dominant dimension. Then ${ }_{R} R$ is a test module for the dominant dimension of the projec. tive modules if and only if each injective projective left module is $\Sigma$-injec. tive.

Proof. $(\Rightarrow)$ It suffices to show that $\bigoplus \Sigma_{\Omega} P_{a}$ is injective, where $P_{a} \cong$ Re for each $\alpha=\Omega$ is an indecomposable injective projective. Further, we may assume that the cardinality of $\Omega$ is $x_{0}$.

Let $0 \rightarrow \bigoplus \Sigma_{\Omega} P_{a} \rightarrow E\left(\bigoplus \Sigma_{\Omega} P_{a}\right)$ be the canonical injection. Since $P_{a}$ is injective, $\bigoplus \Sigma_{\Omega^{\prime}} P_{a}$ is isomorphic to a direct summand of $E\left(\oplus \Sigma_{\Omega} P_{a}\right)$ for each finite subset $\Omega^{\prime}$ of $\Omega$. Moreover, with $E\left(\oplus \Sigma P_{\alpha}\right)$ projective, $E\left(\bigoplus \Sigma_{\Omega} P_{\alpha}\right) \cong \bigoplus \Sigma_{\Gamma} R e_{\beta}$, where each $R e_{\beta}$ is injective. Whereas $\bigoplus \Sigma_{\Omega^{\prime}} P_{\alpha}$ is isomorphic to a direct summand of $\bigoplus \Sigma_{\Gamma} R e_{\beta}$ for each finite subset $\Omega^{\prime}$ of $\Omega, P_{\alpha} \cong R e_{\beta}$ for infinitely many $\beta \in \Gamma$. Thus, $\bigoplus \Sigma_{\Omega} P_{\alpha}$ is injective. 
$\Leftrightarrow$ For ${ }_{R} P$ projective we have ${ }_{R} P \cong \bigoplus \Sigma_{\Omega} R e_{a}$. We consider a minimal injective resolution $0 \rightarrow R e_{a} \rightarrow E_{1 a} \rightarrow \cdots \rightarrow E_{n} a \rightarrow \cdots$ for each $a=\Omega$. Then, with dom $\operatorname{dim}_{R} R \geq n$,

$$
0 \rightarrow \bigoplus \sum_{\Omega} R e_{\alpha} \rightarrow \bigoplus \sum_{\Omega} E_{1 \alpha} \rightarrow \cdots \rightarrow \bigoplus \sum_{\Omega} E_{n a}
$$

provides the desired portion of a minimal injective resolution of $\bigoplus \Sigma R e_{\alpha}$.

Note that the sufficiency part of the proof depended only on the dominant dimension of the projectives being nonzero.

Corollary 1.2. If $R$ is a left perfect ring of positive left dominant dimension and ${ }_{R} R$ is a test module for ${ }_{R} \mathcal{P}$, then $R$ is semiprimary.

Proof. In this setting $E\left({ }_{R} R\right)$ is $\Sigma$-injective. Then, by Corollary 3.4 of [3], $R$ satisfies a.c.c. on left annulets. But then by Proposition 4.1 of [3], $R$ is semiprimary.

Corollary 1.3. A ring $R$ is left self-injective, left perfect and has ${ }_{R} R$ as a test module for $R^{\mathfrak{P}}$ if and only if $R$ is $Q F$.

Proof. For such a ring every projective left module is injective and so, the ring is $Q F$ (see [3]).

In [3] Faith points out that a.c.c. on left annulets of $R$ does not imply $\Sigma$-injectivity of $E\left({ }_{R} R\right)$, although the converse does hold. We provide a case where this is true.

Proposition 1.4. Let $R$ be a left perfect ring of positive left dominant dimension. Then, $\mathfrak{Q}_{\mathrm{nn}_{R}}\left(E\left(_{R} R\right)\right)=\mathbb{Q}_{\mathrm{nn}_{R}}(R)$.

Proof. Let $M$ be a subset of $E\left({ }_{R} R\right) \cong \bigoplus \Sigma_{\Omega} R e_{\alpha}$. Set $M_{\alpha}=\rho_{\alpha}(M) \subseteq R e_{\alpha}$, where $\rho_{a}: E\left({ }_{R} R\right) \rightarrow R e_{a}$ is the canonical projection. Then, $\{r \in R: r M=0\}$ $=\left\{r \in R: r\left(\cup_{\Omega} M_{a}\right)=0\right\}$.

Corollary 1.5. Let $R$ be left perfect and $\operatorname{dom} \operatorname{dim}_{R} R \neq 0$. Then the following are equivalent:

(1) ${ }_{R} R$ is a test module for ${ }_{R} \mathcal{P}$;

(2) $R$ satisfies a.c.c. on left annulets;

(3) For each left ideal I there corresponds a finitely generated sub. ideal I' having the same right annihilator as $I$.

Proof. In light of (1.4) this is a restatement of Proposition 3.3 of [3].

2. The minimal injective cogenerator as a test module. First we note that rings in which codom $\operatorname{dim}_{R} g \neq 0$ have the same $\Sigma$-injectivity property as noted in the previous section. 
Proposition 2.1. Let $R$ be a left perfect ring for which codom $\operatorname{dim}_{R} 9 \neq$ 0 . Then, each injective projective left module is $\Sigma$-injective.

Proof. Theorem 3.1 of [2] states that codom $\operatorname{dim}_{R} \mathfrak{g}=\operatorname{dom}_{\operatorname{dim}_{R}} \mathcal{P}$. Consequently, this is immediate from the remark following (1.1).

In establishing our next theorem we shall make use of the following observation.

Proposition 22. If $R$ is a left perfect ring and ${ }_{R} P$ is an injective projective module which is I-projective, then ${ }_{R} P$ is $\Sigma$-injective. For $R$ perfect the converse also holds.

Proof. It suffices to show that $\bigoplus \Sigma_{\Omega} R e$ is injective for some infinite index set $\Omega, R e$ an indecomposable injective projective left $R$-module. For any infinite set $\Gamma, \Pi_{\Gamma} R e$ is projective and so, isomorphic to $\bigoplus \Sigma_{\Delta} R e_{a}$ for some infinite set $\Delta$. Suppose that only finitely many of the $R e_{a}^{\text {'s }}$ are isomorphic to $R e$, say $n$ in all. The module $\Pi_{\Gamma} R e$ has a submodule isomorphic to $\bigoplus \Sigma^{n+1} R e$, so $\bigoplus \Sigma_{\Gamma} R e_{a}$ has a direct summand isomorphic to $\bigoplus \Sigma^{n+1} R e$. This is impossible and so infinitely many of the $R e_{a}$ 's are isomorphic to $R e$. Thus, $\bigoplus \Sigma_{\Gamma} R e_{a}$ has a direct summand of the form $\bigoplus \Sigma_{\Omega} R e$ with $\Omega$ infinite.

For $R$ perfect, every nonzero module has a nonzero socle. We again let $R e$ be an indecomposable injective projective left $R$-module and consider $\Pi_{\Omega} R e, \Omega$ infinite. Every simple submodule of $\Pi_{\Omega} R e$ is isomorphic to $R e^{\prime} s$ unique simple submodule. Hence, $\Pi_{\Omega} R e \cong \bigoplus \Sigma_{\Gamma} R e$ and $\Pi_{\Omega} R e$ is projective.

So as to relate the codominant dimension of the minimal injective cogenerator ${ }_{R} \mathcal{U}$ to the codominant dimension of an arbitrary injective module, we shall apply the next result.

Proposition 2.3. Let $R$ be left perfect with codom $\operatorname{dim}_{R} 9 \neq 0$ and codom $\operatorname{dim}_{R} \mathcal{U} \geq n$. Then, if $\cdots \rightarrow P_{n} \rightarrow \cdots \rightarrow P_{1} \rightarrow{ }_{R} \mathcal{U} \rightarrow 0$ is a minimal projective resolution of ${ }_{R} \mathrm{U}, P_{i}, 1 \leq i \leq n$, generates the corresponding ith term in the minimal projective resolution of an arbitrary injective left $R$ module.

Proof. By Theorem 3.1 of [2], (1.1) and (2.1), we note that the hypothesis of (1.2) is satisfied. Thus, $R$ is perfect. For each injective ${ }_{R} E$,

$$
{ }_{R} E \cong E\left(\oplus \sum_{\Omega_{1}} \frac{R e_{1}}{J e_{1}}\right) \oplus \cdots \oplus E\left(\oplus \sum_{\Omega_{m}} \frac{R e_{m}}{J e_{m}}\right)
$$


where $e_{1}, \ldots, e_{m}$ belong to a basic set of primitive idempotents for $R$. We shall establish (2.3) for $E\left(\bigoplus \Sigma_{\Omega_{1}} S\right)$, where $S \cong R e_{1} / J e_{1}$.

First observe that $E(\bigoplus \Sigma S)$ is isomorphic to a direct summand of $\Pi_{\Omega_{1}} E(S)$. Next, let $P_{1 n} \rightarrow \cdots \rightarrow P_{11} \rightarrow E(S) \rightarrow 0$ be the first $n$ terms in a minimal projective resolution of $E(S)$.

By (2.1) and (2.2),

$$
\prod_{\mathbf{\Omega}_{1}} P_{1 n} \rightarrow \cdots \rightarrow \prod_{\mathbf{\Omega}_{1}} P_{11} \rightarrow \prod_{\mathbf{\Omega}_{1}} E(S) \rightarrow 0
$$

are the first $n$ terms in a projective resolution of $\Pi E(S)$. Moreover, the $i$ th term in a minimal projective resolution of $E\left(\bigoplus \Sigma_{\Omega} S\right)$ is isomorphic to a direct summand of $\Pi_{\Omega_{1}} P_{1 i}, 1 \leq i \leq n$. Also, such a summand is of the form $\bigoplus \Sigma_{\Gamma} R e_{\beta}$ with each $R e_{\beta}$ isomorphic to a direct summand of $P_{1 i}$. Thus, $P_{1 i}$, and so $P_{i}$, generates $\bigoplus \Sigma_{\Gamma} R e_{\beta}$.

Combining (2.1) and (2.3) along with the results of the previous section we have the following characterization of when the minimal injective cogenerator ${ }_{R} \mathrm{U}$ is a test module.

Theorem 2.4. Let $R$ be left perfect and of positive left codominant dimension. Then the following are equivalent:

(1) ${ }_{R}$ U is a test module for ${ }_{R} 9$.

(2) Each injective projective left module is $\Sigma$-injective.

(3) Each injective projective left module is I-projective.

(4) $R$ has a.c.c. on left annulets and $E\left({ }_{R} R\right)$ is projective.

(5) ${ }_{R} R$ is a test module for $R^{\mathcal{P}}$ and $\operatorname{dom} \operatorname{dim}_{R} R \neq 0$.

Proof. $(1) \Leftrightarrow(2)$. This is (2.1) and (2.3).

(1), (2) $\hookrightarrow(3)$. By Theorem 3.1 of [2], (1.1) and (1.2), we have sufficiency. Necessity is (2.2).

$\Leftrightarrow(4)$. This equivalency is given by Theorem 3.1 of [2], (1.4) and Proposition 3.3 of [3].

$\Leftrightarrow(5)$. See (1.1).

3. When codominant and dominant dimension are equivalent. First we restate the main result of [2], replacing the long tedious proof given there by a greatly clarified version.

Theorem 3.1. Let $R$ be left perfect. Then codom $\operatorname{dim}_{R} \mathfrak{g} \geq n$ if and only if $\operatorname{dom} \operatorname{dim}_{R} \mathcal{P} \geq n$. 
Proof. $(\Rightarrow)$ We induct on $n$. Let ${ }_{R} P$ be projective, and suppose that codom $\operatorname{dim}_{R} 9 \geq k+1$ and that $\operatorname{dom} \operatorname{dim}_{R} \mathcal{P} \geq k$. Consider

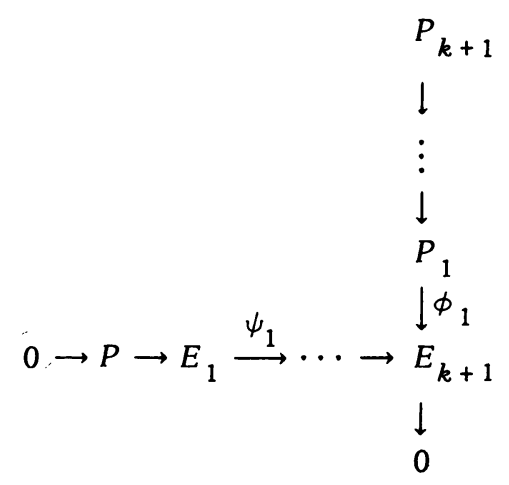

where the row is a minimal injective resolution of ${ }_{R} P$ and the column a minimal projective resolution of $E_{k+1}$. Since $\operatorname{Hom}_{R}\left({ }_{-}, M\right)$ and $\operatorname{Hom}_{R}\left(N,{ }_{-}\right)$are exact functors whenever ${ }_{R} M$ is injective and ${ }_{R}^{N}$ is projective, the above diagram results in the commutative diagram given by Figure 1 , in which all the columns are exact and the first $k+1$ rows are exact and the last row semi-exact. To show that $E_{k+1}$ is projective it will suffice to show that the identity map $\iota \in \operatorname{Hom}_{R}\left(E_{k+1}, E_{k+1}\right)$ has a preimage under $\operatorname{Hom}_{R}\left(E_{k+1}, P_{1}\right) \rightarrow$ $\operatorname{Hom}_{R}\left(E_{k+1}, E_{k+1}\right)$, since in such a case $E_{k+1}$ will be isomorphic to a submodule of $P_{1}$. Using Figure 1 we diagram chase $\iota=\operatorname{Hom}_{R}\left(E_{k+1}, E_{k+1}\right)$ to some $f_{k+1}=\operatorname{Hom}_{R}\left(P, P_{k+1}\right)$ as follows:

$$
\begin{aligned}
& f_{k+1} \rightarrow f_{k} \psi_{0} \\
& \uparrow \\
& f_{k} \rightarrow \text {. } \\
& \uparrow_{f_{2} \rightarrow f_{1} \psi_{k-1}} \\
& \uparrow \\
& f_{1} \rightarrow \psi_{k} \\
& \uparrow
\end{aligned}
$$

Case 1. $f_{i} \psi_{k-i} \neq 0, i=1, \cdots, k$. In this case consider 


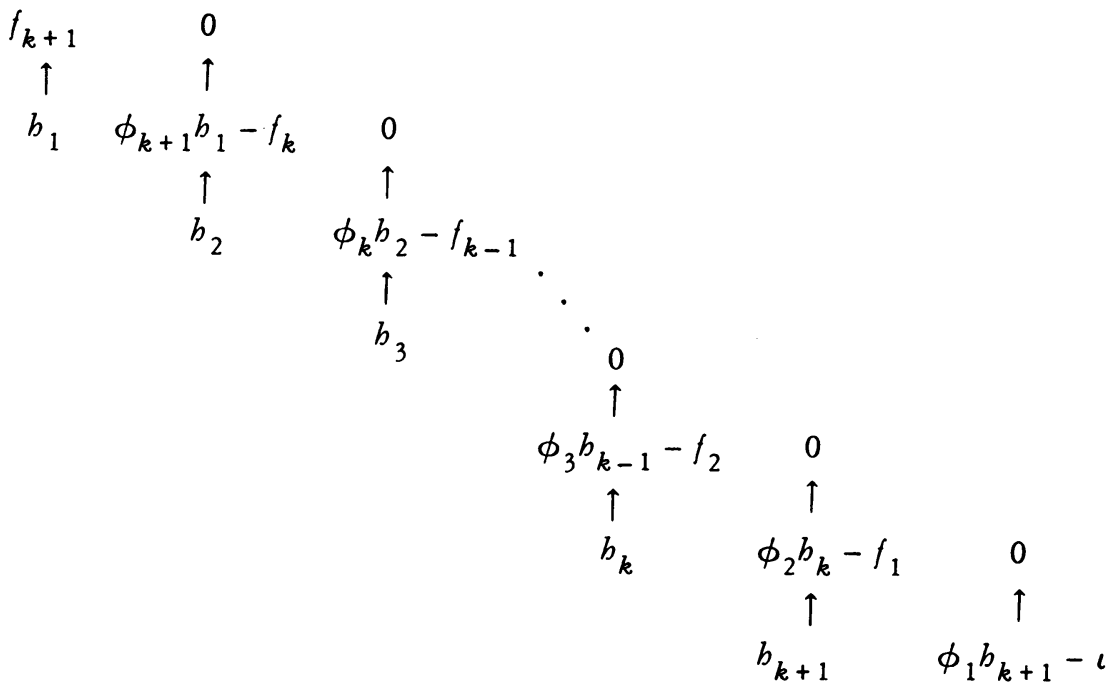

Since $\left(\phi_{1} h_{k+1}-\imath\right) \psi_{k}=0, \phi_{1} h_{k+1} \psi_{k}=\psi_{k}$. But if $\operatorname{Ker} \phi_{1} h_{k+1} \neq 0$, then $\operatorname{Im} \psi_{k} \cap \operatorname{Ker} \phi_{1} h_{k+1} \neq 0$, as $\operatorname{Im} \psi_{k}$ is essential in $E_{k+1}$. This is impossible, so $h_{k+1}$ is monic. Thus, $E_{k+1}$ is isomorphic to a submodule of $P_{1}$ and so, is projective.

Case 2. $f_{i} \psi_{k-i}=0$ for some $1 \leq i \leq k$. Let $l$ be the minimal such $i$. Then we are back in the situation for Case 1 with $k=l-1$.

$\Leftrightarrow$ This is the dual of the sufficiency proof.

For those rings for which the dimensions, respectively, of the injectives and left projectives are positive, the theories of codominant dimension and dominant dimension coincide.

Proposition 3.2. Let $R$ be left perfect and $\operatorname{dom} \operatorname{dim}_{R} \mathfrak{P} \neq 0$. Then, codom $\operatorname{dim}_{R} \mathcal{U}=\operatorname{dom} \operatorname{dim}_{R} R$.

Proof. If not, then by (1.1), (2.1) and (2.4), codom $\operatorname{dim}_{R} \mathfrak{g}=0$.

Corollary 3.3. If $R$ is left perfect and $\operatorname{dom} \operatorname{dim}_{R} \mathcal{P} \neq 0$, then codom $\operatorname{dim}_{R} \mathcal{U}=$ codom $\operatorname{dim} \mathcal{U}_{R^{\prime}}$, where $\mathcal{U}_{R}$ is the minimal injective cogenerator for $\prod_{R}$.

Proof. As noted in (1.2), $R$ must be semiprimary. But then by [5, Theorem 10], right and left dominant dimensions are equal.

To throw slightly more light on those rings for which $\operatorname{dom} \operatorname{dim}_{R} \mathscr{P} \neq 0$, we make the following observations.

Proposition 3.4. Let $R$ be left perfect and $\operatorname{dom} \operatorname{dim}_{R} R>0$. Then, the projective covers of injective left modules are direct sums of finitely gener. ated injectives. 
Proof. For a projective cover $\bigoplus \Sigma R e_{a} \rightarrow{ }_{R} E \rightarrow 0$, we note that each $R e_{\alpha}$ is a submodule of an injective $R e_{\beta}$. Considering

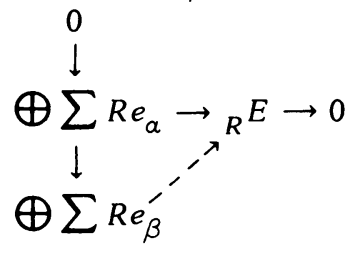

we note that $\bigoplus \Sigma R e_{\alpha}$ is a direct summand of $\bigoplus \Sigma R e_{\beta}$ and, hence, of the desired form.

Corollary 3.5. Let $R$ be left perfect and $\operatorname{dom} \operatorname{dim}_{R} R>0$. Then, if ${ }_{R} U$ is finitely generated or if the injective indecomposable projectives are $\Sigma$. injective, codom $\operatorname{dim}_{R} \mathcal{U} \neq 0$.

Proposition 3.6. Let $R$ be perfect and codom $\operatorname{dim}_{R} \mathbb{U} \neq 0$. Then, $R$ has an injective projective faithful left ideal.

Proof. Let $R e$ be an indecomposable projective. Then,

$$
\begin{aligned}
& 0 \rightarrow R e \rightarrow E(\operatorname{Soc} R e) \cong E(R e) \\
& \because \oplus \sum_{B}^{\uparrow \phi} R e_{\beta}
\end{aligned}
$$

where $\phi$ is the projective cover mapping, can be completed with a monic map. Since $R e$ is finitely generated, $\operatorname{Soc}(R e)$ is isomorphic to a submodule of $\bigoplus \Sigma_{B}{ }^{\prime} R e_{\beta}$ for some finite subset $B^{\prime} \subseteq B$. For a given minimal submodule $S$ of $R e$, there exists $\beta^{\prime} \in B^{\prime}$ such that $S \cong S_{\beta^{\prime}} \leq R e_{\beta^{\prime}}$ and $\phi\left(S_{\beta^{\prime}}\right) \cong S_{\beta^{\prime}}$. Thus, the composition $\rho \phi: \bigoplus \Sigma_{B} R e_{\beta} \rightarrow E(S)$, where $\rho: E(\operatorname{Soc} R e) \rightarrow E(S)$ is the natural projection, does not annihilate the canonical image of $S_{\beta}$. On the other hand, $\bigoplus \Sigma_{B} R e_{\beta}=P \oplus P^{\prime}$, where the restriction of $\rho \phi$ to $P$ provides a projective cover and $P^{\prime}$ is annihilated by $\rho \phi$. Hence, $S$ is isomorphic to a submodule of $P$, and so there exists an injective $\operatorname{Re}_{\beta}$ with $S$ as a submodule. Consequently, $R$ has an injective left ideal which contains a copy of each minimal left ideal of $R$.

Proposition 3.7. Let $R$ be left perfect, and $\operatorname{dom} \operatorname{dim}_{R} R>0$ or codom $\operatorname{dim}_{R} \mathcal{U}>0$. Then, $\operatorname{dom} \operatorname{dim}_{R} \mathcal{P} \neq 0$ if and only if each injective projective left module is $\Sigma$-injective.

Proof. $(\Rightarrow)$ If $R e$ is injective and not $\Sigma$-injective, then $\operatorname{dom} \operatorname{dim} \bigoplus \Sigma_{\Omega} R e=$ 0 for infinite $\Omega$.

$(\longleftarrow)$ See (2.4). 


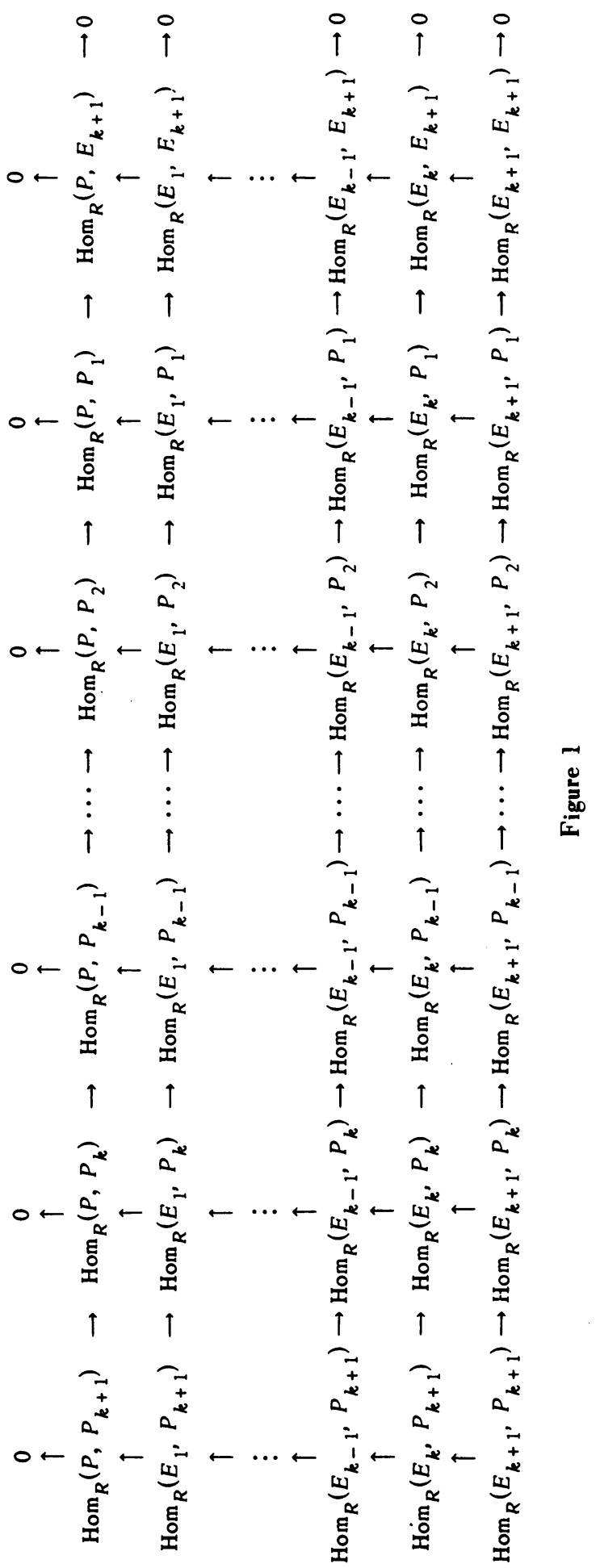




\section{REFERENCES}

1. H. Bass, Finitistic dimension and a homological generalization of semiprimary rings, Trans. Amer. Math. Soc. 95 (1960), 466-488. MR 28 \#1212.

2. G. Eerkes, Codominant dimension of rings and modules, Trans. Amer. Math. Soc. 176 (1973), 125-139. MR 47 \#3455.

3. C. Faith, Rings with ascending condition on annihilators, Nagoya Math. J. 27 (1966), 179-191. MR 33 \#1328.

4. T. Kato, Rings of dominant dimension $\geq 1$, Proc. Japan Acad. 44 (1968), 579-584. MR 38 \#4525.

5. B. Müller, Dominant dimension of semi-primary rings, J. Reine Angew. Math. 232 (1968), 173-179. MR 38 \#2175.

DEPARTMENT OF MATHEMATICS, VIRGINIA POLYTECHNIC INSTITUTE AND STATE UNIVERSITY, BLACKSBURG, VIRGINIA 24060 